\title{
ACUICULTURA DE PARGO LA MANCHA LUTJANUS GUTTATUS (STEINDACHNER, 1869) EN COSTA RICA DENTRO DE UN ENFOQUE ECOSISTÉMICO
}

\author{
Ángel Herrera-Ulloa ${ }^{1 *}$, Jonathan Chacón-Guzmán ${ }^{1}$, Gerando Zúñiga-Calero ${ }^{2}$, \\ Óscar Fajardo y Ricardo Jiménez-Montealegre ${ }^{2}$
}

\begin{abstract}
RESUMEN
La sobreexplotación pesquera y la búsqueda de alternativas ante la pobreza crónica han llevado a explorar el campo de la maricultura; por medio de un enfoque ecosistémico se abrieron dos procesos paralelos: una estrategia e investigación en laboratorio para el levantamiento larval de peces marinos tropicales y una estrategia de comanejo para desarrollar el cultivo de peces marinos tropicales. El pargo la mancha muestra gran potencial de producción de larvas y posterior cultivo en el mar. No mostró respuestas positivas a la inducción por medio de hormonas, el desarrollo de un ambiente adecuado para su reproducción espontánea permitió tener desoves frecuentes y de calidad. La fase de levantamiento larval mostró contratiempos por el tamaño reducido de las larvas de pargo la mancha, el manejo en tanques apropiados y el uso de enriquecedores permitieron mejorar los procesos y llegar a incrementar la producción de alevines. El enfoque ecosistémico permitió abrir los espacios necesarios para la operación de un proyecto piloto. La granja se creó con un enfoque productivo, pero también para turismo rural.
\end{abstract}

Palabras claves: Pargo la mancha, maricultura, enfoque ecosistémico, comanejo.

\begin{abstract}
Overfishing and the search for alternatives to it, led us to the explore mariculture as an option using an ecosystem-based approach through two parallel processes: an in-laboratory research strategy for larval rearing of tropical marine fish, and a strategy of co-management to develop tropical marine fish aquaculture. The spotted rose snapper shows great potential for larvae production and cage culture. This species showed no responses to hormone induction; however, the set up of a suitable environment allowed frequent, high-quality spontaneous spawnings. The larval rearing phase presented problems due to the small size of the larvae; although, management in appropriate tanks and the use of enrichments improved the processes, increasing the production of juveniles. This ecosystem approach allowed the operation of a profitable farm pilot project with a productive approach, but with rural tourism in mind as well.
\end{abstract}

Keywords: Spotted rose snapper, mariculture, ecosystem-based approach, co-management.

1 Parque Marino del Pacífico, Puntarenas, Costa Rica, email: fherrera@una.ac.cr*

2 Escuela de Ciencias Biológicas, Universidad Nacional, Heredia, Costa Rica.

3 Departamento de Acuicultura, Instituto Costarricense de Pesca y Acuicultura, San José, Costa Rica.

Recibido 20-V-2009

Aceptado 05-X-2009

DOI: http://dx.doi.org/10.15359/revmar.1.11

Rev. Mar. y Cost. ISSN 1659-455X. Vol. 1. 197-213, Diciembre 2009. 


\section{INTRODUCCIÓN}

La Organización de las Naciones Unidas para la Pesca y la Agricultura (FAO, 2009a), en relación con la producción pesquera y acuícola global, advierte que es necesario aplicar prácticas pesqueras responsables de forma mucho más generalizada, ya que se ha afectado la distribución de las especies tanto marinas como de agua dulce.

Las pesquerías en pequeña escala (pesca desde la costa o en botes en la zona costera o en agua dulce) hacen una importante pero subvalorada contribución a las economías de algunos de los países más pobres. La evaluación y manejo de dichas pesquerías es usualmente inadecuada o ausente y se menosprecia su potencial como motor para el desarrollo y el cambio (Andrew et al. 2007). Los conflictos con el recurso pueden ser disminuidos, el manejo mejor implementado y los recursos mejor administrados, cuando los pescadores y otros interesados se encuentran más involucrados con el manejo de los recursos y los derechos de acceso son distribuidos más equitativa y efectivamente (Pomeroy, 1995).

En relación con la sobreexplotación pesquera, Pikitch et al. (2004) señalan la necesidad de una visión de manejo holística, usando el manejo ecosistémico, con un objetivo centrado en la salud del ecosistema marino y las especies que allí viven, consiguiendo así actuar sobre los impactos de las pesquerías en las especies en peligro de extinción y sobre las áreas protegidas. Con un manejo ecosistémico, se puede planear, desarrollar y manejar y las pesquerías, en forma tal, que se conduzcan las múltiples necesidades y deseos de la sociedad, sin comprometer las necesidades de las futuras generaciones, beneficiando todos los bienes y servicios proveídos por los ecosistemas marinos (FAO, 2003).

La extracción y producción acuícola de Costa Rica (Figura 1) es pequeña si se compara con otras naciones, y en comparación con otras actividades económicas del país, además con muchos altibajos; las pesquerías demersales fueron importantes hasta el año 2000 cuando disminuyó casi en un 50\%, incrementándose la pesquería de tiburón, básicamente dedicada al "aleteo". Esta pesquería decayó en casi un $60 \%$ en el 2004 cuando se incrementó la regulación y control sobre la flota pesquera en su mayoría taiwanesa. En el 2002, la acuicultura había pasado a ser el principal rubro, siendo la exportación su oferta principal. A pesar de las cifras anteriores, la actividad pesquera costarricense juega un papel especialmente importante con respecto a la generación de mano de obra en las zonas costeras, no solo como alternativa primaria de trabajo para sectores marginales, sino porque constituye la pesca artesanal de pequeña escala, la principal fuente de abastecimiento de productos marinos para el consumo interno. $\mathrm{La}$ 
Figura 1

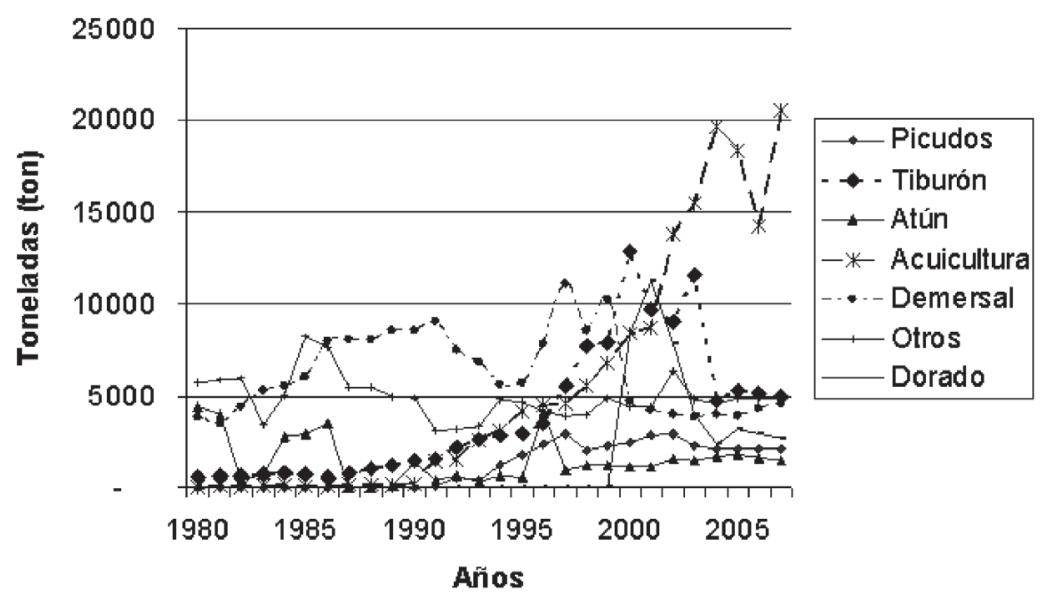

Estadísticas de pesca y acuicultura para Costa Rica, 1980-2007 (fuente: FAO 2009b). Costa Rica fisheries and aquaculture statistics, 1980-2007 (source: FAO 2009b).

importancia estratégica de los recursos pesqueros debe visualizarse en términos de valoración social, dado que con base en criterios económicos, como se señaló previamente, resulta poco relevante (Herrera et al. 2009 in rev.).

La sobreexplotación pesquera es parte de los problemas que provocan la pobreza existente en las zonas costeras de Costa Rica. Esta pobreza es considerada como pobreza crónica (Hulme et al. 2001), donde además del problema de ingreso, se da una privación de los sistemas de salud, aislamiento, falta de educación, falta de atención y falta de seguridad.

La búsqueda de alternativas ante la pobreza crónica en la zona costera de Costa Rica ha llevado a explorar el campo de la maricultura, con los problemas de la carencia de una legislación apropiada, ausencia de una zonificación de áreas marinas y la falta de políticas. En los años 90 se iniciaron varios proyectos de producción de semilla y cultivo del pargo la mancha (Lutjanus guttatus), con un enfoque experimental que permitió conocer los aspectos básicos de su comportamiento y potencial para su reproducción en cautiverio y cultivo masivo en jaulas flotantes, pero a pesar de contribuir con las primeras bases hacia la promoción de esta nueva actividad productiva y alternativa para el sector, su desarrollo fue infructuoso. Herrera et al. (2008) consideran que ha existido poca efectividad de las organizaciones públicas con asociaciones aisladas e insuficientes, careciendo de investigación, 
información, promoción, control y política.

En el 2002 se inició un nuevo esfuerzo para el desarrollo de la maricultura en Costa Rica con un enfoque ecosistémico, como estrategia para integrar una posible industria de maricultura, dentro del ecosistema marino, promoviendo el desarrollo sostenible, la equidad, la resiliencia de la conexión entre el sistema social y ecológico. Se fomentó la investigación para la producción en laboratorio de una especie marina con potencial económico y se apoyó a grupos organizados de pescadores artesanales para mejorar su forma de vida tradicional. El proyecto fue desarrollado por el Parque Marino del Pacífico, organización del Ministerio de Ambiente, Energía y Telecomunicaciones (MINAET), con el apoyo de la Universidad Nacional (UNA), sumándose posteriormente la Asociación de Pesca y Cultivo de Isla Venado, el Instituto Costarricense de Acuicultura (INCOPESCA) y el International Cooperation and Development Fund (ICDF) de la República Democrática de Taiwán. Retomando trabajos desarrollados en los años 90, se decidió seguir la investigación con la especie pargo la mancha (Lutjanus guttatus) (Steindachner, 1869).

El pargo la mancha es una especie demersal, que habita en arrecifes costeros, hasta unos $30 \mathrm{~m}$ de profundidad, en general solitario o en pequeños grupos, pero ocasionalmente forma grandes cardúmenes; los juveniles viven en estuarios y bocas de ríos (Allen, 1995). Se distribuye desde el Golfo de California, México, hasta el Perú. Es la especie demersal de mayor importancia comercial del pacífico costarricense (Vargas, 1999). Gutiérrez y Durán (1999) demostraron que era factible económicamente cultivar pargo la mancha para generar empleo y riqueza en forma sostenible, pero que era necesario implementar un laboratorio de producción de alevines. Los aspectos antes señalados, sumados a su comportamiento y desempeño, motivaron la investigación y desarrollo de esta especie con fines de cultivo. El desarrollo comercial de pargo la mancha está en fase piloto, existen muchos problemas biotecnológicos por resolver, esperándose desarrollarlo a niveles similares a las de otras especies marinas, tales como Rachycentrum canadum (Benetti et al. 2008b), Mugil cephalus, Chanos chanos, Coryphaena hippurus o $\mathrm{Pa}$ ralichthys lethostigma (Lee and Ostrowski, 2001).

El presente trabajo analiza una estrategia de manejo e investigación científica para desarrollar el cultivo sostenible de peces marinos tropicales en Costa Rica, enfocado a sectores sociales de escasos recursos.

\section{METODOLOGÍA}

El proceso dentro de un enfoque ecosistémico tiene dos fases principales y sobrepuestas para implementar 


\section{Figura 2}

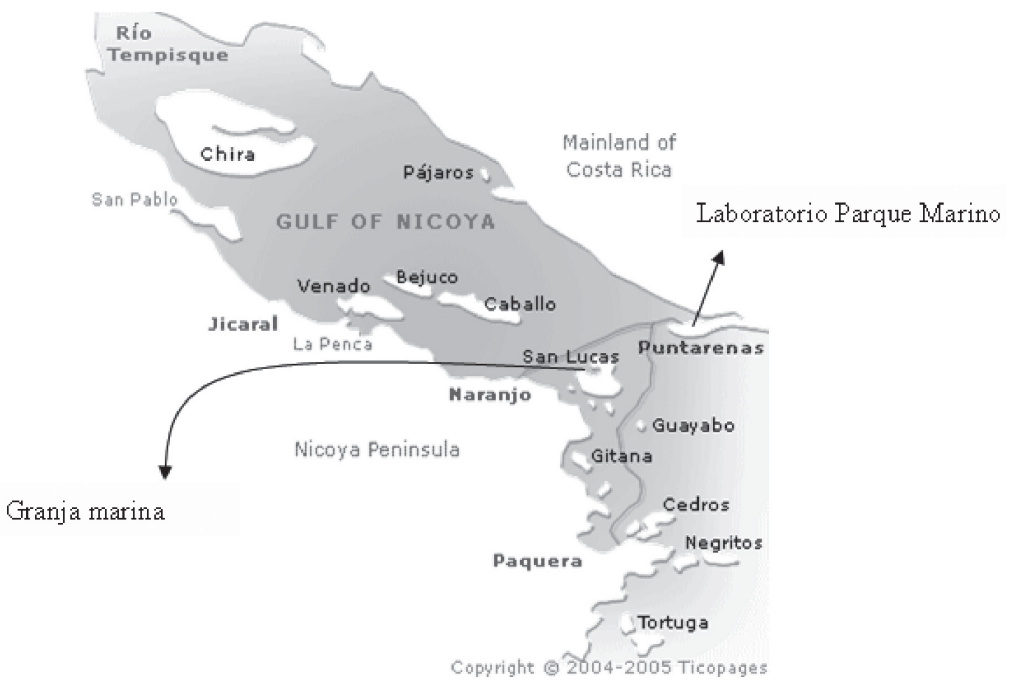

Ubicación del Parque Marino del Pacífico y la granja de cultivo de pargo la mancha, Golfo de Nicoya, Costa Rica.

Location of Parque Marino del Pacifico and spotted rose snapper farm, Gulf of Nicoya, Costa Rica.

en el Golfo de Nicoya (Figura 2), la estrategia e investigación en laboratorio para el levantamiento larval de peces marinos tropicales, y la estrategia de comanejo para desarrollar el cultivo de peces marinos tropicales.

Estrategia de investigación en laboratorio para el levantamiento larval de peces marinos tropicales: En el año 2002 se transfirió la biotecnología de acuicultura de la Universidad Nacional (UNA) al Parque Marino del Pacífico, y se asignó personal científico como un mecanismo para propiciar avances en la investigación y la gestión tecnológica en maricultura. El personal de la UNA opera con un esquema de administración pública, y el personal del Parque Marino opera con un esquema de empresa privada, existiendo diferencias organizacionales. Por lo anterior, para que los dos sistemas de trabajo pudieran interactuar en un mismo espacio se creó un modelo organizacional (Figura 3) de interacción; por medio de mecanismos organizacionales se fijó un objetivo específico sobre el que se focaliza todo el personal. Así, toda la producción de alimento vivo (fitoplancton y zooplancton), las investigaciones en nutrición, la maduración de los reproductores, la larvicultura y precría de alevines, y la calidad de aguas, debían desarrollarse y ser medidas con base en la producción sostenible de alevines, cualquiera que fuera la especie.

En el 2002 y por dos años, dos familias de reproductores se mantu- 
Figura 3

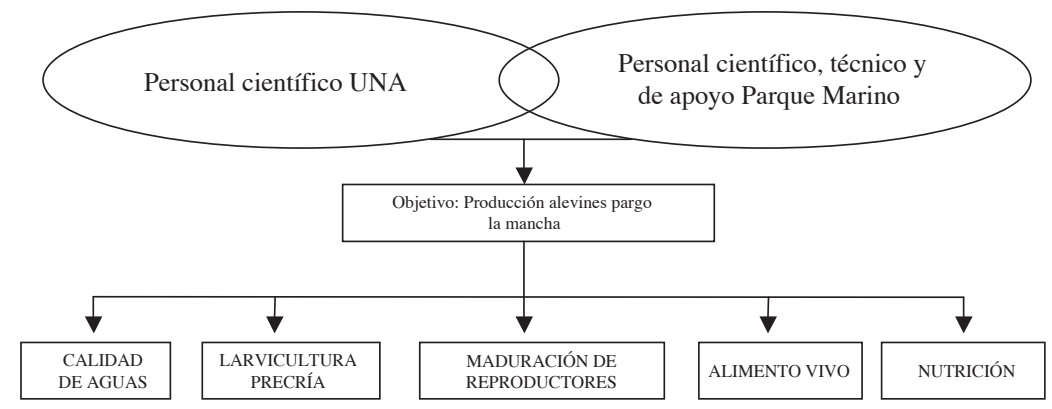

Modelo de interacción laboral y organigrama productivo para la investigación y desarrollo de alevines de pargo la mancha.

Research and development organizational scheme for spotted rose snapper juvenile production.

vieron en sistemas de flujo continuo con un $100 \%$ de recambio directo, en tanques circulares de fibra de vidrio de $20 \mathrm{~m}^{3}$, con una densidad aproximada de $2 \mathrm{~kg} / \mathrm{m}^{3}$ y una relación de dos machos por cada hembra. La cantidad de peces por tanque varió con el tiempo, con un promedio de 12 reproductores en cada tanque. Se llevó el registro de parámetros fisicoquímicos (salinidad, oxígeno disuelto y $\mathrm{pH}$ ), y tres veces al día se observó el comportamiento en los sistemas de flujo continuo por espacio de 30 minutos durante 289 días.

En el año 2004, los reproductores se trasladaron a un área cerrada y aislada, seis tanques de $20 \mathrm{~m}^{3}$ en fibra de vidrio fueron colocados con sistemas de recirculación cerrada y filtros biológicos, con un recambio cerrado diario del $200 \%$ y un aporte de agua nueva del $5-10 \%$ diario. Se mantuvo en promedio la misma cantidad de peces por tanque, pero se varió la relación a dos hembras por macho. La alimentación se basó en gusanos poliquetos ( $25 \%$ del peso total fresco), camarones $(25 \%)$, calamares $(25 \%)$ y pescado (25\%).

Se indujeron desoves con Gonadotropina Coriónica, de aplicación subcutánea a una dosis de 900 UI/ kg por hembra en dos dosis, con una diferencia de 24-26 horas entre ambas y una sola dosis de $300 \mathrm{UI} / \mathrm{kg}$ por cada macho, aplicada en el momento de la segunda dosis de las hembras.

Con las primeras producciones sostenidas de huevos, se iniciaron los levantes larvales. La larvicultura se realizó en tanques circulares de fibra de vidrio de $6 \mathrm{~m}^{3}$. Se aplicaron en promedio 500 litros diarios de $T e$ traselmis chui con densidades entre las 100 y $200 \mathrm{cel} . / \mathrm{ml}$; se mantuvieron rotíferos (Brachionus rotundiformis) enriquecidos con Rotimac ${ }^{\circledR}(0.5 \mathrm{~g} /$ millón de rotíferos), entre los 5 y 20 individuos $/ \mathrm{ml}$ y se alimentó con artemia a razón de 1 individuo/ml. Se aplicó la primera alimentación al segundo día posteclosión basada en 
microalgas y rotíferos, los que se mantuvieron hasta el día 20. A partir del día 15-16 y hasta el día 30 se aplicaron nauplios de artemia, y del día 25 al 35 se aplicó artemia adulta a razón de 2 individuos/larva/día, la cual funcionó como un complemento alimenticio.

El período de destete inició entre los días 30 y 32 , lo cual depende de la temperatura, ya que a temperaturas bajas $25-26^{\circ} \mathrm{C}$, el metabolismo de las larvas es menor y el desarrollo se prolonga hasta el día 32, mientras que a temperaturas mayores $28-29^{\circ} \mathrm{C}$ su metabolismo se acelera y su desarrollo es más rápido iniciándose en el día 30. Se aplicó una mezcla de alimento fresco basado especialmente en camarón y pescado. El destete se logró en un período de ocho días y los alevines se trasladaron a tanques circulares de fibra de vidrio de $12 \mathrm{~m}^{3}$ para su etapa de finalización en laboratorio (del día 38-40 al día 55-60). Desde el día 1 al 4 no se realizó recambio de agua, del 5 al 25 se recambió un 30\%, del 25 al 40 un $100 \%$ y del 40 al 60 un $150 \%$.

Estrategia de comanejo dentro de un enfoque ecosistémico: En el año 2002, con la apertura del Parque Marino del Pacífico, grupos organizados de pescadores iniciaron presión para desarrollar cultivos marinos, se evaluaron las experiencias y errores en materia de acuicultura en el país, y se decidió implementar un modelo de comanejo ecosistémico, como una forma de descentralización de las ins- tituciones y con el objetivo de obtener una producción sostenible de alevines para propiciar una industria que ofertara dicha producción, enfatizando en el empoderamiento de los usuarios del sector pesquero artesanal.

Se consolidó y desarrolló una operación de investigación en los laboratorios de producción marina del Parque Marino del Pacífico con personal de dicha institución y de la Universidad Nacional. Desde un inicio hubo interés y presión de grupos organizados de comunidades pesqueras artesanales del Golfo de Nicoya, y fue esto lo que motivó que en el año 2005 se conformara una red de instituciones estatales involucradas en el sector pesquero artesanal y conformada por el Instituto Costarricense de Pesca y Acuicultura (INCOPESCA), el Instituto Mixto de Ayuda Social (IMAS) y la Universidad Nacional (UNA), con la participación de comunidades pesqueras; se creó además una alianza con el International Cooperation and Development Fund de la República de Taiwán, que apoyó la fase de laboratorio e impulsó y financió la fase de campo. En la Figura 4 se esquematiza la interrelación de cada parte en relación con el objetivo principal (producir alevines) y en relación con los distintos actores involucrados.

En el año 2006, el Parque Marino obtuvo tres concesiones marinas. Tres grupos de pescadores fueron seleccionados y se inició la construcción de la primera granja marina en conjunto con la Asociación de Pesca y Cultivo 


\section{Figura 4}

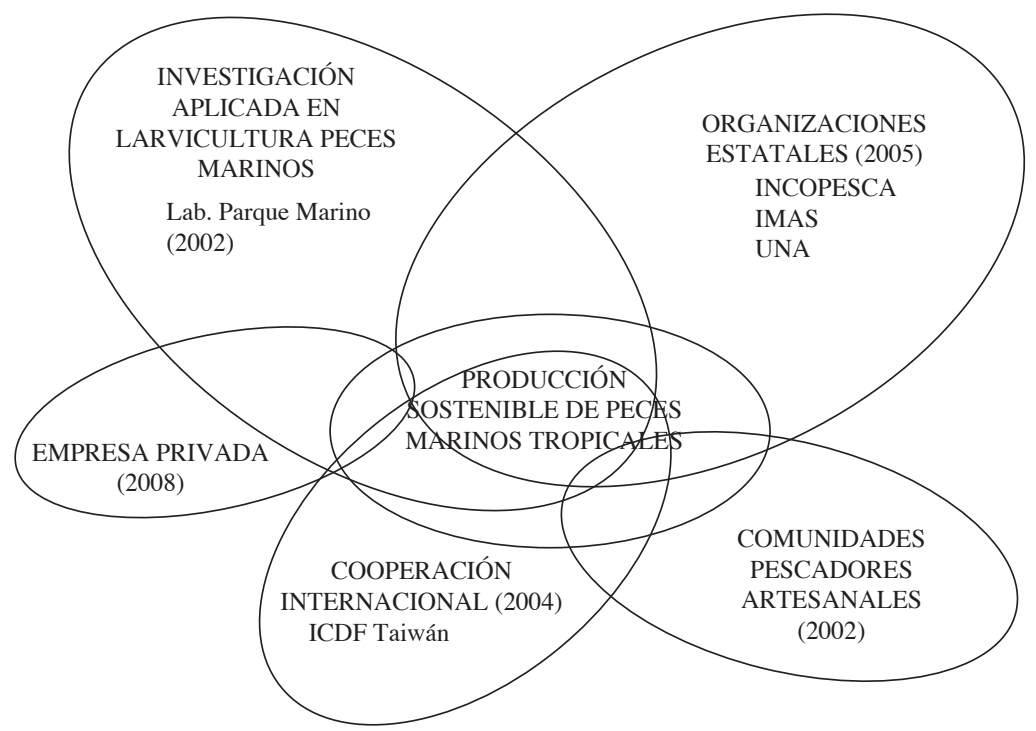

Esquema del proceso de comanejo para la producción sostenible de peces marinos tropicales.

Diagram of the comanagement process for sustainable tropical marine fish production.

de Isla Venado. Los sitios de cultivo fueron seleccionados con base en un estudio biológico, ambiental, social y económico, siendo el primer sitio para el establecimiento de jaulas de cultivo la zona de Playa Leona $\left(9^{\circ} 55^{\prime} 33^{\prime \prime} \mathrm{N}\right.$ y $84^{\circ} 55^{\prime} 32^{\prime}$ 'W), Golfo de Nicoya. Diez jaulas flotantes fueron construidas con marco de hierro galvanizado y andamios de madera de $6 \times 6 \mathrm{~m}$ con una red de nailon de 3,5 metros de profundidad, $0,5 \mathrm{~m}$ de red saliente y $3 \mathrm{~m}$ sumergida ( $108 \mathrm{~m}^{3}$ de volumen total). Para la flotación de cada jaula se utilizaron 8 recipientes plásticos de 189 litros. Cada jaula se construyó independiente una de la otra, amarradas entre sí por cuerda de 1". El sistema de anclaje consistió en 10 bloques de concreto, dos de cinco toneladas y ocho de dos toneladas. El ancla- je se sujetó a una boya de liberación de tensión y de esta a las jaulas y plataformas flotantes con una cuerda de 1".

Se construyeron tres plataformas flotantes, la primera de $13 \times 6 \mathrm{~m}$ con una estructura en tubo de hierro galvanizado, piso de madera y una pequeña casa (cocina) de $4 \times 4 \mathrm{~m}$. Dos plataformas se hicieron de $6 \times 6 \mathrm{~m}$, con casas del mismo tamaño que funcionarían como bodegas y dormitorios. Se colocaron en línea con la corriente principal y se comunicaron por pequeños puentes de madera.

Seis jaulas fueron llenadas con alevines de laboratorio (seis mil en total); los individuos de tres de las jaulas se alimentaron con un pienso artificial de prueba tipo extrusado fabricado por la división de concentra- 
dos de la Corporación PIPASA, con una formulación para peces marinos que varió de 40 a $42 \%$ de proteína; y los individuos de las otras tres jaulas se alimentaron con alimento fresco (camarón y sardina). El control de crecimiento se llevó a cabo por un período de doce meses, se midieron los parámetros de oxígeno y salinidad, ocasionalmente la temperatura, con el fin de garantizar la estabilidad del sistema y del crecimiento de los juveniles.

En el 2008, el proceso se amplió a la empresa privada, con el afán de escalar las producciones y mejorar las posibilidades futuras de desarrollo del cultivo en el país, por medio de inversión privada al sector.

\section{RESULTADOS}

Maduración natural y reproducción espontánea en cautiverio: Las condiciones permitieron mantener reproductores en buen estado de salud e inclusive llevarlos a su maduración; los desoves inducidos con hormonas no fueron exitosos y no se logró obtener desoves espontáneos constantes durante los dos primeros años, con dos excepciones, que en promedio no sobrepasaron los 100.000 huevos por desove, y no sobrepasaron los quince días de cultivo.

Los promedios de las variables fisicoquímicas se mantuvieron normales según las condiciones reportadas para las aguas de la parte externa del Golfo de Nicoya y con

\section{Cuadro 1}

Promedios de las principales variables fisicoquímicas tomadas durante el mantenimiento de reproductores de pargo la mancha en dos tanques de $20 \mathrm{~m}^{3}$ con flujo abierto (años 2002-2003).

\section{Table 1}

Averages of the main physico-chemical variables in two $20 \mathrm{~m}^{3}$ open flow tanks for rose spotted snapper broodstock.

\begin{tabular}{ccc}
\hline & Tanque 1 & Tanque 2 \\
\hline Temperatura ${ }^{\circ} \mathrm{C}$ & 28.6 & 28.7 \\
\hline $\mathrm{pH}$ & 8.1 & 8.1 \\
\hline Salinidad \%o & 33.8 & 33.8 \\
\hline
\end{tabular}

muy poca variación entre los dos tanques (Cuadro 1). La variación de la temperatura durante el día osciló entre 1 y $2^{\circ} \mathrm{C}$.

El comportamiento de los pargos en uno de los tanques (tanque 1) fue normal (peces en el fondo del tanque, natación normal y sin agitación aparente) durante 228 días (79\%) de los 289 días; mostraron condiciones de agrupación o posible cortejo (peces agrupados de la mitad de la columna 
del agua hacia arriba, por lo general con movimiento de persecución en forma circular) durante 32 días (11\%) y de estrés durante 29 días $(10 \%)$. En el otro tanque (tanque 2), la observación tuvo una duración de 227 días, el comportamiento normal fue durante 179 días (79\%), durante 39 días (16\%) fue un comportamiento de agrupación y mostraron estrés durante 9 días $(4 \%)$.
Al trasladar los peces reproductores al área aislada y con sistemas recirculados, se pudo lograr que los peces desovaran espontáneamente (Figura 5). A medida que los reproductores de origen silvestre permanecieron en cautiverio y con el mejoramiento de estas condiciones, la producción de huevos aumentó anualmente (Figura 6), alcanzando un promedio de 2.160.000 huevos/mes en los primeros meses del 2008. La fertilidad pro-

\section{Figura 5}

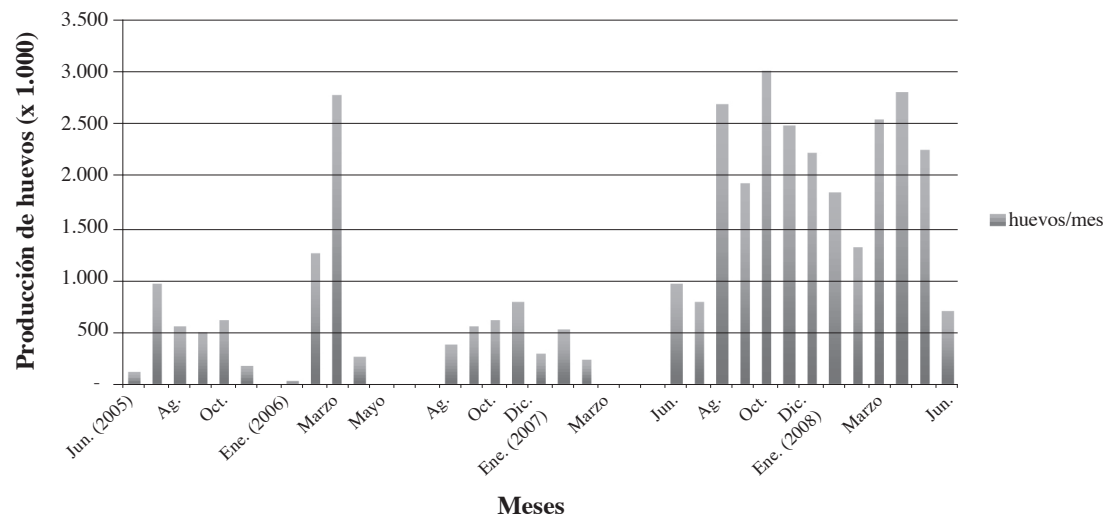

Producción de huevos de pargo la mancha (Lutjanus guttatus) en el Parque Marino del Pacífico, Costa Rica (junio 2005 a junio 2008).

Spotted rose snapper spawn production (Lutjanus guttatus) at Parque Marino del Pacifico, Costa Rica (June 2005-June 2008).

medio se estimó en un $90 \%$, mientras que el porcentaje de eclosión de los huevos fértiles fue del $85 \%$.

Los promedios de las variables fisicoquímicas de tres tanques de los nuevos sistemas mostraron muy poca diferencia con respecto a las variables de los tanques con flujo abierto (temperatura $29.5{ }^{\circ} \mathrm{C}$, oxígeno di- suelto $5.4 \mathrm{mg} / \mathrm{L}, \mathrm{pH} 8.7$ y salinidad $31.2 \%$, Cuadro 1).

Larvicultura: Los procesos de producción de larvas fueron optimizados durante los primeros años en función de obtener un protocolo de alimentación que permitiese la sobrevivencia de las larvas. Producto de esta optimización se desencadenaron, entre julio del 2005 y julio del 


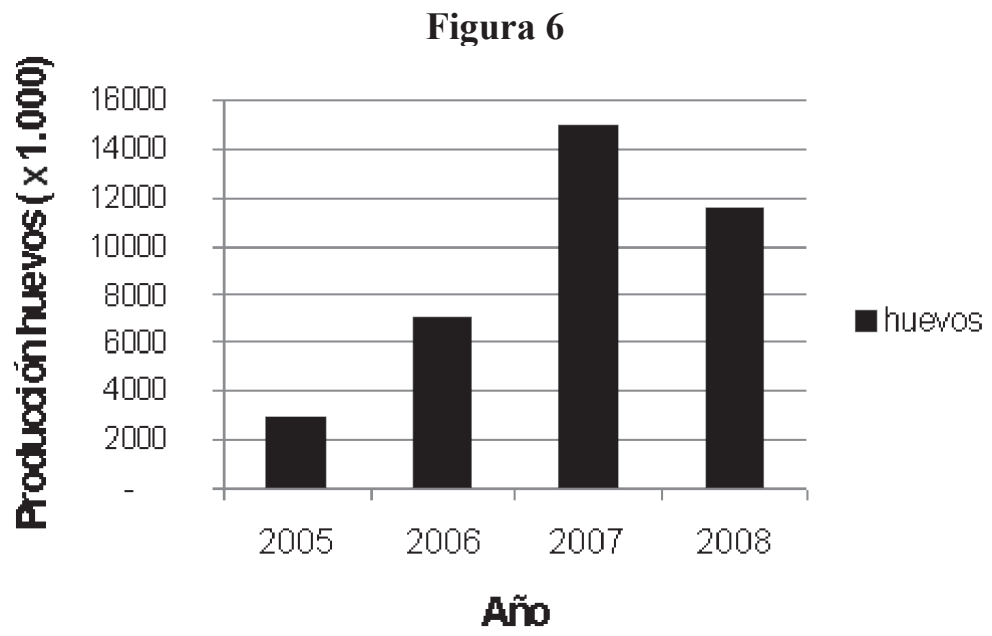

Producción anual de huevos de pargo la mancha (L. guttatus) en el Parque Marino del Pacífico, Costa Rica.

Annual production of spotted rose snapper (L. guttatus) spawn at Parque Marino del Pacifico, Costa Rica.

2008, alrededor de 180.000 alevines de pargo la mancha. La fase de alimentación plantónica larvaria consistió de microalgas (Tetraselmis sp.), rotíferos (Brachionus rotundiformis) $\mathrm{y}$ artemia (nauplios y adultos), mientras que para el destete y precría se utilizó especialmente alimento fresco suministrado cinco veces al día ad libitum. Con la optimización de la tecnología se han obtenido mejores resultados, pasando de una producción de 10.000 alevines en el 2005 a cerca de 70.000 en el 2007 (Figura 7).

La granja marina: La estructura de la granja marina se diseñó con base en el sistema de anclaje, jaulas de cultivo y plataformas. Toda la estructura fue donada, el $75 \%$ por el ICDF y el $25 \%$ por medio del IMAS, a la Asociación de Pesca y Cultivo de Isla Venado, que se encargó de la operación y mantenimiento, así como del manejo de la producción. Los resultados en el crecimiento de pargos se truncaron por un abrupto rompimiento de relaciones de Costa Rica con Taiwán, lo que provocó la salida de ICDF. Además, la estructura se deterioró a partir del tercer año de su instalación.

Las variables ambientales y fisicoquímicas del agua durante los períodos de cultivo se mostraron estables sin variaciones importantes que pudieran haber afectado el crecimiento de los peces. Durante los primeros 18 meses de cultivo se cosecharon para la venta directa en el mercado de mariscos de Costa Rica 12.057 ejemplares de pargo la mancha, con un peso total vendido de $4.320 \mathrm{~kg}$, producto de cinco cosechas 
Figura 7

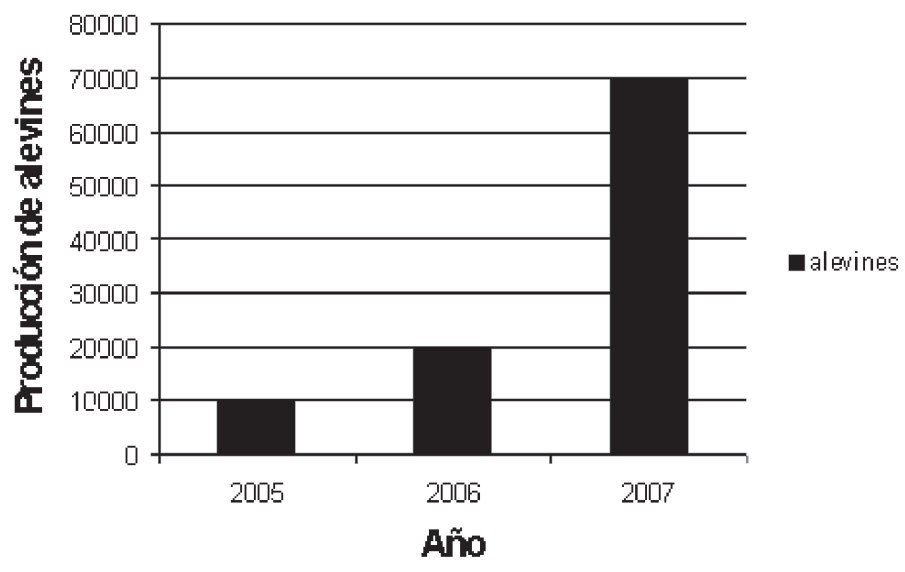

Producción anual de alevines de pargo la mancha (L. guttatus) en el Parque Marino del Pacífico, Costa Rica.

Annual production of spotted rose snapper (L. guttatus) juveniles at Parque Marino del Pacifico, Costa Rica.

parciales. La categoría comercial de venta fue la " $0-1$ ", que indica peces de menos de 1 libra de peso; el promedio de peso de venta fue de $0.36 \mathrm{~kg} /$ individuo. El precio promedio fue de $\$ 3.71 /$ $\mathrm{kg}$, manteniéndose casi constante. Durante este período de prueba, en el cual se optó por producir pocos peces para lograr una mejor adaptación por parte de los pescadores a las medidas de manejo, se logró una utilidad aproximada de $\$ 4.77$ dólares (Cuadro 2); dentro de los costos se incluyen los gastos al día de 4 personas en alimentación y transporte.

Paralelo al cultivo, se abrió un servicio de comidas en el marco de turismo rural, ofreciendo además pesca deportiva en los alrededores de la granja. Después de la salida del ICDF de Taiwán, este negocio se convirtió en el principal ingreso. El negocio mostró utilidades de \$3.105 para la primera estación, y de $\$ 10.980$ para la segunda, generando además empleo (Cuadro 3).

El desarrollo del proyecto encontró un notable vacío institucional, desde reglas claras sobre los procedimientos, hasta la ausencia de una zonificación marina. El operacionalizar el proyecto se hizo mediante procesos informales de coordinación entre las organizaciones institucionales participantes y los sectores pesqueros involucrados.

\section{DISCUSIÓN}

Los reproductores son la base para todo el proceso en la producción 


\section{Cuadro 2}

Producción de pargo la mancha en jaulas flotantes y utilidad (\$) obtenida durante los primeros 18 meses (2005-2006).

\section{Table 2}

Spotted rose snapper production in floating cages and profit (\$) during the first 18 months (2005-2006).

\begin{tabular}{|c|c|c|c|c|c|c|c|c|}
\hline & Categoría & $\begin{array}{c}\text { Venta } \\
\text { (kg) }\end{array}$ & $N^{0}$ peces & $\begin{array}{l}\text { Peso prom. } \\
\text { (kg) }\end{array}$ & $\begin{array}{c}\text { Precio } \\
\text { (\$/kg) }\end{array}$ & $\begin{array}{c}\text { Ingresos } \\
(\$)\end{array}$ & $\begin{array}{c}\text { Costo } \\
\text { (\$) }\end{array}$ & $\begin{array}{c}\text { Utilidad } \\
(\$)\end{array}$ \\
\hline Venta 1 & $0-1$ & 800 & 2.000 & 0.40 & 3.87 & 3.095 & 2.166 & 928 \\
\hline Venta 2 & $0-1$ & 900 & 2.571 & 0.35 & 3.50 & 3.150 & 2.205 & 945 \\
\hline Venta 3 & $0-1$ & 800 & 2.286 & 0.35 & 3.50 & 2.800 & 1.960 & 840 \\
\hline Venta 4 & $0-1$ & 11.20 & 3.200 & 0.35 & 3.50 & 3.920 & 2.744 & 1.176 \\
\hline Venta 5 & $0-1$ & 700 & 2.000 & 0.35 & 4.20 & 2.940 & 2.058 & 882 \\
\hline Total & & 4.320 & 12.057 & & & 15.905 & 11.133 & 4.771 \\
\hline Promedio & & 1.207 & 2.411 & 0.36 & 3.71 & 3.181 & 2.227 & 954 \\
\hline
\end{tabular}

\section{Cuadro 3}

Ingreso y utilidades obtenidas en la granja de cultivo de pargo la mancha producto de turismo rural (junio 2007-junio 2008).

\section{Table 3}

Income and profit derived from rural tourism activities at the spotted rose snapper farm (June 2007-June 2008).

\begin{tabular}{lcccccc}
\hline & Anual & $\begin{array}{c}\text { Ingreso } \\
\text { Bruto (\$) }\end{array}$ & \% Costos & Costos (\$) & \% Utilidad & $\begin{array}{c}\text { Utilidad } \\
\text { (\$) }\end{array}$ \\
\hline $\begin{array}{l}\text { Visitantes Temporada Baja } \\
\text { (Abril - Nov.) }\end{array}$ & 640 & 9.412 & 66.70 & 6.306 & 33.3 & 3.105 \\
\hline $\begin{array}{l}\text { Visitantes Temporada Alta } \\
\text { (Dic. - Marzo) }\end{array}$ & 2.400 & 31.373 & 65.00 & 20.392 & 35 & 10.980 \\
\hline Total & & 40.785 & & 26.698 & & 14.085 \\
\hline
\end{tabular}

de peces, y los resultados obtenidos durante los dos primeros años demostraron que se debían ajustar las variables fisicoquímicas y ambientales del agua y del entorno. Durante el período de observación de reproductores de pargo la mancha en tanques de flujo abierto, se determinó que la calidad del agua variaba según las condiciones ambientales externas al laboratorio, y que ello no permitía el control óptimo para mantener una situación constante en el tiempo. Los días que los peces mostraron estrés coincidieron con algún evento externo, ya sea contaminación sónica o mala calidad del agua.

La realización de pruebas con hormonas es un procedimiento común en acuicultura, realizado para 
lograr la sincronización en los desoves de varias hembras y así obtener una mayor producción de huevos. En este trabajo se concluyó que el pargo la mancha no responde de manera óptima a este método, por lo que la búsqueda de un ambiente apropiado para desoves espontáneos pasó a ser la meta principal, permitiéndoles a los reproductores "decidir" el momento justo para su reproducción, y determinando a la vez los requerimientos mínimos especialmente en calidad de agua, cantidad y tipo de alimentación, y condiciones generales del área de maduración. Los cambios realizados y sus resultados exitosos corroboran lo indicado por Benetti y Alarcón (2000), quienes afirman que el manejo de los lotes de reproductores es crítico para el desarrollo de cualquier proyecto de producción en acuicultura, y que de ello dependerá la calidad de los huevos, larvas y alevines a producir.

La producción de huevos observada (Figura 5) confirmó la asincronía en los desoves de pargos, lo que coincide con Rojas (1997), quien señala los meses de marzo y octubre como los de reproducción de esta especie en el Golfo de Nicoya; además, se da una disminución de los desoves en relación directa con la baja ambiental de la temperatura para los meses de diciembre a febrero.

El levante larval es el mayor obstáculo en la producción de larvas de pargo la mancha; dadas las reducidas tallas de las larvas y la poca amplitud de sus bocas, se debió lograr una producción controlada de alimento vivo de pequeños tamaños, incrementando a su vez los costos de producción. A partir de los desoves espontáneos naturales en espacio cerrado, se optó por realizar los levantes larvales en tanques de $6 \mathrm{~m}^{3}$, utilizando enriquecedores que permitieran complementar el valor nutricional del alimento vivo, resultando en la obtención de las primeras sobrevivencias masivas (hasta 5.000 alevines por tanque), con una tasa de sobrevivencia que varió de 0.1 a $3.5 \%$ del total de huevos desovados, mejorando la producción de huevos año con año (Figura 6). El incremento en la producción de alevines (Figura 7) se debió primero al aumento de la producción de huevos y segundo a las mejoras en los procesos de levantamiento larval, con alimentación diaria de hasta cinco veces y con recambios de agua entre el 100 y $300 \%$ diario, dependiendo de la edad de la larva. Los resultados encontrados refuerzan el potencial de la producción en laboratorio de la especie como ha sido encontrado para la cobia (Benetti et al. 2008a).

La granja marina se diseñó con un objetivo mixto de producción y turismo rural, con un efecto positivo directo en el medio ambiente al disminuir el esfuerzo pesquero, y social al cambiar los pescadores su actividad hacia un cultivo y manejo de un proyecto pequeño sostenible.

La fase de prueba del cultivo determinó que las condiciones ambien- 
tales y fisicoquímicas del agua en el sitio escogido permiten el cultivo comercial del pargo manchado, sin embargo, el manejo por parte de los pescadores no permite obtener resultados concretos sobre las variables que ayudan o desfavorecen la producción. No obstante, esta fase permitió reafirmar que el pargo la mancha es una especie con una demanda insatisfecha, y que representa un precio nacional e internacional muy llamativo para su comercialización (Cuadro 2). La perspectiva de turismo tomó mayor importancia con la finalización de la ayuda por parte de Taiwán, y finalmente permitió la subsistencia del proyecto, así como la generación de mayores ingresos y fuentes de empleo. Chuenpagdee y Jentoff (2007) señalan un proceso para el comanejo en pesquería, que en mucho resume lo realizado en el desarrollo de la granja marina, donde se establecen las condiciones y las directrices, se da la inspiración y la concepción, se da la inicialización y la comunicación, la participación y la preparación, y finalmente, la reflexión y adaptación.

\section{CONCLUSIÓN}

Los primeros intentos para el desarrollo de cultivo comercial de pargo la mancha inician en Costa Rica, y la fase de laboratorio se ha hecho con la participación de la Universidad $\mathrm{Na}$ cional (UNA), el Parque Marino del Pacífico, y con el apoyo posterior del ICDF. El pargo la mancha no mostró respuestas positivas a la inducción por medio de hormonas. El desarrollo de un ambiente adecuado para su reproducción espontánea permitió tener desoves frecuentes y de calidad. La fase de levantamiento larval mostró contratiempos por el tamaño reducido de las larvas de pargo la mancha, pero el manejo en tanques apropiados y el uso de enriquecedores permitió mejorar los procesos y llegar a incrementar la producción de alevines. Los resultados en laboratorio muestran un claro potencial y factibilidad para que el pargo la mancha se pueda desarrollar en cultivos comerciales.

El enfoque ecosistémico formulado permitió desarrollar en campo la fase de crecimiento por medio de la interrelación entre varias instituciones y pescadores, y con estrategia de comanejo con pescadores organizados, abriéndose así los espacios necesarios que permitieron la operación de un proyecto piloto. La granja se creó con un enfoque productivo, pero también para turismo rural, motivado esto por la salida abrupta de la cooperación del ICDF que dejó desfinanciado el proyecto; el componente de turismo rural permitió al proyecto su sobrevivencia, por la presencia de ingresos constantes.

\section{AGRADECIMIENTOS}

A Luis Villalobos, por sus oportunos puntos de vista que colaboraron a mejorar sustancialmente el documento. Al señor Chen Chen Hui y a la ICDF de Taiwán, que dieron el 
apoyo y entusiasmo necesarios para seguir con el proyecto. Al Ministerio de Ambiente, Energía y Telecomunicaciones, que ha apoyado la investigación sobre pargo la mancha, como una potencial alternativa sostenible para poblaciones costeras.

\section{BIBLIOGRAFÍA}

Allen, G. (1995). Lutjanidae. Pp. 1231-1244. En: Fischer, W., F. Krupp, W. Schneider y V. H. Niem (Red. Téc.). Guía FAO para la identificación de especies para los fines de la pesca. Vol. III Pacifico Centro-oriental. FAO, CE, FIS, NORAD. Roma.

Andrew, N., Bené, C., Hall, S., Allison, E., Heck, S. and Ratner, B. (2007). Diagnosis and management of small-scale fisheries in developing countries. Fish fish., 8, 227-240.

Benetti, D., Sardenberg, B., Welch, A., Hoening, R., Refik Orhum, M. and Zink, I. (2008a). Intensive larval husbandry and fingerling production of cobia Rachycentron canadum. Aquac., 281, 22-27.

Benetti, D., Orhun, M., Sardenberg, B., O'Hanlon, B., Welch, A., Hoenig, R., Zink, I., Rivera, J., Denlinger, D., Bacoat, D., Palmer, K. and Cavalin, F. (2008b). Advances in hatchery and growout technology of cobia Rachycentron canadum (Linnaeus). Aquac. Res., 39 (7), 701-711
Benetti, D. and Alarcón, J. (2000). General Prophylaxis and Quarantine of Marine Brood Fish. Tropical Marine Fish Farming. Diciembre. 60-61 p.

Chuenpagdee, R. and Jentoff, S. (2007). Step zero for fisheries co-management: what precedes implementation? Mar. Pol., 31, 657-668.

FAO. (2003). Fisheries Management 2 . The ecosystem approach to fisheries. FAO Technical Guidelines for Responsible Fisheries. No. 4, Suppl. 2. Rome.

FAO. (2009a). El estado mundial de la pesca y la acuicultura, 2008. Departamento de Pesca y Acuicultura. Roma.

FAO. (2009b). Fisheries Department. Data and Statistics Unit. FISHSTATS Plus: Universal Software for fishery statistical time series. Version 2.2. 2000.

Gutiérrez, R. y Durán, M. (1999). Cultivo del pargo la mancha Lutjanus guttatus (Pisces: Lutjanidae) en jaulas flotantes. Uniciencia, 15 (1), 27-34.

Herrera-Ulloa, A., Chacón-Guzmán, J., Guevara-Lizano, J. and Jiménez-Montealegre, R. (2008). Rose Snapper Marine Culture in Costa Rica. Panorama Acuicola Mag., 13 (3), 38-42.

Herrera-Ulloa, A., Villalobos-Chacón, L., Palacios-Villegas, J., Víquez-Portuguez, R. and Oro-Marco, G. (2009 in rev.). Chapter 6. Coastal Fisheries of Costa Rica. 
Hulme, D., Moore, K. and Shepherd, A. (2001). Chronic Poverty: meanings and analytical frameworks. CPCR Working Paper No. 2. Manchester and Birmingham, Chronic Poverty Research Centre. (CPRC website http://www.chronicpoverty.org)

Lee, C. and Ostrowski, A. C. (2001). Current status of marine finfish larviculture in the United States. Aquaculture 200 (1-2), 89-109.

Pikitch, E. K., Santora, C., Babcok, E. A., Bakun, A., Bonfil, R., Conover, D. O., Dayton, P., Doukaki, P., Fluharty, D., Heneman, B., Houde, E. D., Link, J., Livingsgton, P. A., Mangel, M., McAllister, M. K., Pope, J. and Sainsbury, K. J. (2004). Ecosystem-based fi- shery management. Science, 305, 346-347.

Pomeroy, R. (1995). Community based and co-management institutions for sustainable coastal fisheries management in Southeast Asia. Ocean. Coas. Manag., 27 (3), 143-162.

Rojas, M. J. R. (1997). Fecundidad y épocas de reproducción del "pargo la mancha" Lutjanus guttatus (Pisces: Lutjanidae) en el Golfo de Nicoya, Costa Rica. Rev. Biol. Trop., 44 (3), 477-487.

Vargas, M. (1999). Estimación de parámetros biológico-pesqueros para el pargo la mancha Lutjanus guttatus en el Golfo de Nicoya, Costa Rica. Uniciencia, 15-16, 79-84. 